\title{
Educación superior en Trabajo Social en Chile y formación para la intervención en situación de calle. Desafíos desde la evaluación que interventores hacen de sus procesos de práctica pre-profesional
}

\author{
Carlos Alejandro Andrade-Guzmán \\ Ingeniero en Comercio Internacional. Magíster en Gestión y Políticas Públicas \\ Universidad Alberto Hurtado. Santiago, Chile \\ https:/ / orcid.org/0000-0003-4150-032X • caandrade@uahurtado.cl
}

\section{Ignacio Andrés Eissmann-Araya}

Sociólogo. Magíster en Gobierno y Sociedad

Centro de Investigación e Incidencia para el fin de la situación de calle en América Latina-CISCAL y Corporación Moviliza, Santiago, Chile

https://orcid.org/0000-0001-9967-3392 • ieissmann@ciscal.org

Resumen

La práctica pre-profesional es clave en la formación en trabajo social. En este marco, esta investigación buscó identificar la evaluación que los trabajadores sociales chilenos hacen de los aprendizajes intencionados en estas instancias para la intervención en situación de calle. Metodológicamente, en base a un diseño cuantitativo con alcance exploratorio-descriptivo, se invitó a responder un cuestionario online que fue contestado por interventores de la disciplina. Los resultados muestran, en términos generales, altas evaluaciones, pero con dispersión, con interventores evaluando altamente sus aprendizajes mientras que otros no. Esto sugiere que no todos sentirían que han incorporado las mejoras herramientas para la intervención en calle. Se discuten implicancias para la formación como, entre otros, la definición participativa de elementos mínimos que los estudiantes debiesen incorporar en sus prácticas sujeta a permanente revisión. Asimismo, el que existe el desafío de avanzar hacia el fortalecimiento de la colaboración entre la institución en donde se realiza la práctica y la casa de estudios, en pos de contribuir de mejor manera al proceso formativo de los estudiantes de Trabajo Social.

Palabras clave: Trabajo Social; Educación Superior en Trabajo Social; Intervención en Situación de Calle; Práctica Pre-profesional.

Recibido: 15/02/2020|Aprobado: 06/05/2020

(i) (2) Esta obra está bajo una Licencia Creative Commons Atribución-NoComercial-CompartirIgual $\underline{4.0 \text { Internacional. }}$

Procedencia del artículo: Artículo derivado del proyecto de investigación Procesos de Formación en Trabajo Social para la Intervención con Personas en Situación de Calle, conducido desde la ciudad de Boston, Estados Unidos, durante el período 2018-2019, en el marco de los estudios doctorales de sus autores. El estudio se sostuvo con recursos autogestionados.

¿Cómo citar este artículo? / How to quote this article?

Andrade-Guzmán, C.A., y Eissmann-Araya, I.A. (2020). Educación superior en Trabajo Social en Chile y formación para la intervención en situación de calle. Desafíos desde la evaluación que interventores hacen de sus procesos de práctica pre-profesional. Prospectiva. Revista de Trabajo Social e intervención social, (30), $239-258$. doi: 10.25100/prts.v0i30.8931. 
Andrade-Guzmán, y Eissmann-Araya

\title{
Higher Education in Social Work in Chile and Training for Intervention in Situations of Homelessness. Challenges in the Evaluation Done by Practitioners of Students' Pre-Service Processes of Practice
}

\begin{abstract}
Pre-service practice is key in social work training. In this framework, this research sought to identify the evaluation of Chilean social workers of the intended learning in these instances for intervention in a street situation. The methodology, based on a quantitative design with an exploratory-descriptive scope, consisted of an online questionnaire answered by practitioners of the discipline. The results show, in general terms, high scores, but with variances because some practitioners assess student learning highly while others do not. This suggests that not all the practitioners feel that learners have incorporated the best tools for intervening in the domain of homelessness. Implications for training are discussed, such as, among others, the collaborative definition of minimum elements that students should incorporate into their practices, subject to permanent review. Also, there is the challenge of moving towards the strengthening of the collaboration between the institution where the practice is carried out and the house of studies, in order to contribute better to the training process of Social Work students.

Keywords: Social Work; Social Work Higher Education; Intervention in Homelessness; Pre-service Practice.

Sumario: 1. Introducción, 2. Premisas conceptuales y posición paradigmática en esta investigación, 3. Metodología, 4. Hallazgos, 4.1 Apoyo recibido en la práctica preprofesional, 4.2 Comprensión sobre la situación de calle, 4.3 Herramientas para la intervención, 4.4 Contribuciones específicas para la formación en Trabajo Social, 4.5 Comprensión sobre el rol de las condiciones de soporte a la intervención, 5. Conclusiones, 6. Referencias bibliográficas.
\end{abstract}




\section{Introducción}

La Educación en Trabajo Social en Chile, como su profesionalización, está presente desde 1925, cuando se creó la primera Escuela de Trabajo Social en el país y en América Latina (Calderón y Cortés, 2014; González, 2010; Illanes, 2006; Vidal, 2015). En este contexto, desde su inicio el Trabajo Social ha puesto su atención en abordar situaciones relacionadas con la pobreza (Vidal, 2015) y otros fenómenos que hoy se pueden considerar como expresiones de exclusión social.

$\mathrm{Al}$ respecto, cabe relevar que uno de los grupos que es posible reconocer como los que se encuentran en mayor situación de exclusión social, corresponde a las personas en situación de calle o sin hogar. En este marco, el Informe del relator especial sobre vivienda adecuada del año 2015 señala que la falta de vivienda es una crisis mundial de derechos humanos (ONU. Consejo de Derechos Humanos, 2015). Destaca que la falta de vivienda es una violación extrema de los derechos a una vivienda adecuada y a la no discriminación, así como también que puede afectar otros derechos como: a la vida, a la seguridad personal, a la salud, a la familia y a no ser sometidos a un trato inhumano y cruel (ONU. Consejo de Derechos Humanos, 2015).

Los elementos expuestos hablan de la multidimensionalidad y complejidad que rodea el fenómeno de la situación de calle. Esta complejidad, en mayor o menor medida, ha sido reconocida por las intervenciones sociales, las cuales han evolucionado desde el refugio y la asistencia alimentaria a modelos más complejos dirigidos a la entrega de la vivienda y la integración social (Eissmann y Cuadra, 2018;Federación Europea de Organizaciones Nacionales-FEANTSA, 2016; Padget, Henwood\& Tsemberis, 2016; Us Department of housing and urban development office of policy development and research, 2010). En esta línea, esta mayor complejidad implica quelas personas trabajadoras sociales que han de intervenir en torno al fenómeno necesitan llevar adelante intervenciones sociales más integrales, en diálogo con un abordaje que responda a la complejidad del fenómeno. Por consiguiente, el tipo de formación que requieren en sus estudios superiores exige igualmente un mayor nivel de complejidad en sus diferentes componentes. Ello, tanto en el aula como en los espacios de práctica pre-profesional. En este sentido, diversos autores reconocen que la historia del Trabajo Social en Chile tiene diferentes etapas desde su creación en 1925 como profesión, que respondió tanto a sus contextos sociales como a los desafíos inherentes al desarrollo de la educación superior (Salamé y Castañeda, 2009). En este sentido, en las últimas décadas, el contexto del desarrollo de las escuelas de Trabajo Social, en Chile, ha estado marcado por las demandas del Ministerio de Educación en términos de calidad de los servicios educativos, y la alta competencia de las instituciones de educación superior (Salamé y Castañeda, 2009). Como resultado, las escuelas de Trabajo Social comenzaron una revisión y reformulación de sus 
Andrade-Guzmán, y Eissmann-Araya

planes de estudio, donde también surgió la formación basada en competencias como respuesta a las preguntas planteadas sobre la educación profesional (Castañeda y Salamé, 2003), y que a su vez representa una tendencia global que apunta a generar procesos de formación de mayor calidad sin perder la esencia y los objetivos de la disciplina (Duarte y Mora, 2016; López y Chaparro, 2006).

En este contexto, algunos autores afirman que la formación basada en competencias no solo obedece a los requerimientos del mercado laboral, sino que debe guiarse por las demandas planteadas por los problemas sociales, lo que implica el desarrollo de una formación integral (Duarte y Mora, 2016; López y Chaparro, 2006).

Con respecto al contexto de las carreras de Trabajo Social en la educación superior, algunos estudios muestran que, en general, la oferta de carreras ha aumentado en los últimos años, concentrándose principalmente en tres ciudades: Santiago, Valparaíso y Concepción (Saravia, 2015). Sin embargo, respecto a la certificación de calidad de estas carreras, una gran cantidad no se encuentra acreditada (Saravia, 2015).En este sentido, un estudio que compara la relación que existe entre la oferta universitaria y la de los institutos técnicos profesionales muestra que, aunque legalmente el Trabajo Social es exclusivamente universitario, en la práctica se puede ofrecer como oferta académica de institutos profesionales (Aspeé, 2016). A su vez, indica que desde 2016 ha habido un aumento considerable en la oferta de estos institutos en comparación con las carreras universitarias, donde las vacantes de escuelas de universidades tradicionales (y acreditadas con mayor calidad) han tendido a disminuir (Aspeé, 2016; Saravia, 2015). Al respecto, hoy día el Trabajo Social es considerado como un campo altamente fragmentado, lo cual se evidencia al considerar que

en la actualidad existen 107 ofertas de programas universitarios de Trabajo Social; 97 de Servicio Social ofrecidos por Institutos Profesionales; 17 programas de Trabajo Social en Institutos Profesionales; 61 técnicos en Trabajo Social dictados por Centros de Formación Técnica; 50 programas de técnicos en Servicio Social y 94 de técnicos en Trabajo Social dictados por Institutos Profesionales, lo que da un total de 426 ofertas de formación terciaria. (Iturrieta, 2017, p. 17).

En este contexto, tanto en las carreras universitarias como en la oferta de los institutos profesionales o centros de formación técnica, los planes curriculares se centran en generar las competencias de la población estudiantil para comprender e intervenir en diversos fenómenos sociales, utilizando el conocimiento y las metodologías aprendidas durante los años de estudio. Por esta razón, en la mayoría de las carreras profesionales de Trabajo Social, se llevan a cabo prácticas pre-profesionales, cuyo objetivo es que el conjunto de estudiantes adquieran y desarrollen habilidades profesionales, aplicando también lo que han aprendido en las aulas. Al respecto, cabe señalar que, a pesar de la relevancia de este componente formativo, no se advierte la presencia de estudios que, 
centrándose en el caso chileno, se hayan orientado a observar cómo las personas formadas en Trabajo Social evalúan los aprendizajes promovidos por los espacios de práctica preprofesional para la intervención en el campo específico de la situación de calle.

Es dentro de este marco que se sitúa este estudio, orientándose a responder a la siguiente pregunta: ¿cómo los trabajadores sociales chilenos evalúan los aprendizajes promovidos dentro de sus espacios de práctica pre-profesional para la intervención en el ámbito de la situación de calle?

En este escenario, el trabajo se estructura en las siguientes secciones: la primera, conformada por esta introducción; en la segunda se exponen las premisas conceptuales que han orientado este estudio, así como también el posicionamiento paradigmático desde el cual se ha llevado adelante; en la tercera se expone la metodología con la que se ha desarrollado la investigación; en la cuarta se presentan los hallazgos y, finalmente, una quinta sección de conclusiones en la que se exponen algunos elementos iniciales de discusión, las limitaciones identificadas en este trabajo e implicancias para la educación superior en Trabajo Social en Chile desde una perspectiva crítica para la intervención en situación de calle.

\section{Premisas conceptuales y posición paradigmática en esta investigación}

La posición paradigmática de este estudio respecto al Trabajo Social es crítica (Healy, 2001). Entiende la conexión entre la disciplina y los derechos humanos (Al-Nashif, 2018; De Robertis, 2018; Rubilar, 2018; Staub-Bernasconi, 2016) como indivisible. En este marco, a su vez entiende que la educación en Trabajo Social debe concebirse al servicio de los derechos humanos (ONU. Centro de Derechos Humanos, 1995) y, por tanto, la disciplina del Trabajo Social constituye una manera de crear condiciones para contribuir al ejercicio de los derechos y para aportar a la superación de la exclusión social. Desde esta perspectiva, la formación en Trabajo Social debe cumplir con los más altos estándares de calidad al preparar a los futuros trabajadores que se involucrarán en iniciativas orientadas a la transformación social. Esto es extensible a la intervención con personas en situación de calle, grupo que a nivel internacional ha sido reconocido como uno de los prioritarios de la acción del Trabajo Social (Belcher, Pecukonis\& Knight, 2011). Dicho esto, este estudio entiende a las personas en situación de calle o sin hogar como un grupo que sufre la constante violación de sus derechos humanos. Debido a esto, los estudiantes de Trabajo Social necesitan contar con la mejor preparación en su proceso de educación superior para la acción interventiva en este campo. Al respecto, cabe relevar que, en adelante, se hará mención a "profesionales del Trabajo Social" o "Trabajadoras/es sociales" en sentido genérico. Ello, en tanto en este estudio se han recogido igualmente las experiencias de 
personas que en su educación superior han seguido la carrera de nivel técnico en el área. Asimismo, se adherirá a la concepción de disciplina en su sentido amplio como generadora de conocimiento $\mathrm{y}$, en este contexto, relacionada pero diferente del concepto de profesión; a su vez, este trabajo busca contribuir a la formación profesional en términos de generación de conocimiento relativo a la educación superior en Trabajo Social ${ }^{1}$.

Dentro de este marco y debido a la conexión entre la disciplina y la realización de derechos, en este caso, de las personas sin hogar o en situación de calle, este estudio entiende que la formación en Trabajo Social "debe proveer a los estudiantes la oportunidad de adquirir conocimientos, habilidades, métodos de intervención, valores éticos y estándares para la práctica profesional" (Shera \& Bogo, 2001, p. 199). Al hacerlo, la educación en Trabajo Social busca combinar los conocimientos proporcionados tradicionalmente en el aula con las prácticas (Birkenmaier, Wilson, Berg-Weger, Banks \& Hartung, 2003), en este caso, pre-profesionales. En este contexto, tradicionalmente, el componente de práctica ha sido visto como "el "corazón" de la educación en Trabajo Social” (Robertson, 2013, p. 99). Así, la práctica pre-profesional sería como en el caso de la formación en investigación un espacio fundamental para la dinamización de aprendizajes (Rosas-Escamilla y Maldonado-Maldonado, 2018). En este sentido, sería un componente clave de formación al servicio de preparar a las y los estudiantes para que desempeñen su rol en las estrategias de intervención social (Parola, 2020; Wayne, Raskin \& Bogo, 2010).

En función de su relevancia, desde este trabajo se entiende que la posibilidad de acompañar los espacios formativos de práctica pre-profesional con una reflexión crítica, revisando las propias concepciones paradigmáticas que se tienen respecto al poder, la desigualdad y el privilegio desde un marco de justicia social y una perspectiva anti opresiva (Das \& Carter, 2014; Parola, 2020), se vuelve relevante en la formación de futuros y futuras trabajadores sociales. Al hacerlo, se entiende que la función de la supervisión es crucial debido, entre otras, a la retroalimentación que quienes supervisan pueden proporcionar como un mecanismo de mejora al aprendizaje de las y los estudiantes (Andrews \& Harris, 2017; Beddoe, Ackroyd, Chinnery \& Appleton, 2011; Kourgiantakis, Sewell\& Bogo, 2019; Puig-Cruells, 2020). En palabras de Ghiraldelli (2020), “la supervisión permite potenciar el pensamiento, las capacidades y competencias profesionales. También favorece el aprendizaje, impide el acomodo acrítico a las situaciones, o sea, persigue interrogarse, actuar de forma reflexiva y contribuye a la generación de conocimiento" ( $p$. 46). Esto, en tanto generadora de espacios para la reflexión crítica (Beddoe et al., 2011) y en pos de cuestionar, entre otras, las propias posiciones paradigmáticas, teóricas y metodológicas para intervenir, de manera similar a como es promovido en la formación en investigación (Gorostiaga, 2017).

1 Para profundizar en una reflexión sobre disciplina y profesión en Trabajo Social en Chile, se sugiere revisar, entre otros, el trabajo de Toledo (2004). 


\section{Metodología}

\section{Alcance del estudio y muestra}

El estudio es exploratorio-descriptivo. El diseño muestral fue no probabilístico de tipo estratégico (o por conveniencia) (Cea, 2001), convocando a participar voluntariamente a trabajadoras y trabajadores sociales chilenos que cumplían con los siguientes criterios: 1) contar con educación superior en Trabajo Social, 2) haber obtenido su título en una institución de educación superior chilena y, 3) contar con al menos una experiencia de práctica pre-profesional en el área de situación de calle durante su formación de pregrado. En el caso de las y los encuestados con más de una experiencia práctica en este campo, se les pidió responder el cuestionario tomando como referencia la última práctica preprofesional.

Si bien el muestreo por conveniencia no requiere de un listado poblacional previo, cabe relevar que, para contar con una referencia de potenciales participantes invitados a responder el instrumento de este estudio, se recopilaron listas de identificación de diferentes trabajadoras y trabajadores sociales que desarrollaron práctica pre-profesional en calle. Para ello, se contó con el apoyo de las siguientes instituciones: Corporación Moviliza, Hogar de Cristo, Corporación Catim y Universidad Alberto Hurtado. Estas listas permitieron hacer una aproximación a nivel nacional de, por un lado, profesionales con grado de licenciatura y título universitario en Trabajo Social y, por otro, con formación profesional o técnica, pero sin el grado de licenciatura. Tomando estas listas como marco de referencia, se envió el cuestionario por correo electrónico a antiguos estudiantes de Trabajo Social. Complementando esta estrategia, el cuestionario se subió a redes sociales como LinkedIn y Facebook.

En este contexto, los datos se recopilaron durante el mes de octubre de 2018. Cabe señalar que, buscando aumentar el número de encuestados, se enviaron correos semanales de seguimiento a las personas potenciales a participar. Esta estrategia permitió arribar a un número final de 64 cuestionarios. Dentro de este marco, se recolectó un número limitado de personas con formación técnica, razón por la cual los datos producidos por este estudio fueron trabajados usando dos categorías de referencia: "Título profesional y Licenciatura en Trabajo Social" y "Educación Profesional o Técnica (sin Licenciatura)". Cabe relevar que todas las personas que contestaron el instrumento manifestaron su acuerdo con participar en el estudio, dando su consentimiento informado al comienzo del cuestionario. Considerando estos elementos, la Tabla 1 presenta los estadísticos descriptivos de la muestra: 
Andrade-Guzmán, y Eissmann-Araya

Tabla 1. Estadísticos descriptivos de la muestra

\begin{tabular}{|c|c|c|}
\hline Variables & Porcentaje & Media \\
\hline \multicolumn{3}{|l|}{ Género } \\
\hline Femenino & 81.2 & \\
\hline Masculino & 18.8 & \\
\hline \multicolumn{3}{|l|}{ Tipo de Educación } \\
\hline Título profesional y Licenciatura en Trabajo Social & 67.2 & \\
\hline Educación Profesional o Técnica (sin Licenciatura) & 32.8 & \\
\hline \multicolumn{3}{|l|}{ Régimen de estudios } \\
\hline Diurno & 67.2 & \\
\hline Vespertino & 32.8 & \\
\hline \multicolumn{3}{|l|}{ Región de estudios } \\
\hline Antofagasta & 6.3 & \\
\hline Araucanía & 6.3 & \\
\hline Biobío & 9.4 & \\
\hline Los Lagos & 6.3 & \\
\hline Metropolitana & 48.4 & \\
\hline Valparaíso & 23.4 & \\
\hline \multicolumn{3}{|l|}{ Situación mientras estudiaba } \\
\hline Solo estudiando & 37.5 & \\
\hline Estudiando y trabajando en diferentes temas de Trabajo Social & 15.6 & \\
\hline Trabajando en diversas actividades & 46.9 & \\
\hline \multicolumn{3}{|l|}{ Apoyo económico durante los estudios } \\
\hline Beca del Estado & 10.9 & \\
\hline Beca interna de la institución & 4.7 & \\
\hline Beca y préstamo & 20.3 & \\
\hline Préstamo & 39.1 & \\
\hline Sin apoyo económico & 25 & \\
\hline \multicolumn{3}{|c|}{ Experiencia previa en situación de calle (anterior a la práctica pre-profesional) } \\
\hline Sin experiencia previa & 56.3 & \\
\hline Experiencia de trabajo & 3.1 & \\
\hline Experiencia como voluntario/a & 23.4 & \\
\hline Práctica previa & 12.5 & \\
\hline Distintas experiencias previas & 4.7 & \\
\hline \multicolumn{3}{|l|}{ Momento de la carrera en que desarrolló la práctica pre-profesional } \\
\hline Después de concluir todos los cursos & 3.1 & \\
\hline Después de concluir todos los cursos y en paralelo al proceso de tesis & 21.9 & \\
\hline Durante los primeros años de formación & 6.3 & \\
\hline Durante los últimos años de formación & 29.7 & \\
\hline En una etapa intermedia & 39.1 & \\
\hline \multicolumn{3}{|l|}{ Tipo de organización en la que desarrolló la práctica pre-profesional } \\
\hline Organización de la sociedad civil & 93.7 & \\
\hline Municipio & 6.3 & \\
\hline Duración promedio de la práctica (en meses) & & 6 \\
\hline Tiempo promedio semanal dedicado a la práctica (en horas) & & 18.7 \\
\hline
\end{tabular}

Fuente: elaboración propia 


\section{Instrumento y análisis de datos}

38 indicadores fueron incluidos en la versión final del instrumento luego de un proceso de pre testeo. De ellos, 21se utilizan para responder la pregunta de investigación en este artículo. Estos se agrupan en cinco dimensiones: "Apoyo académico a la práctica pre-profesional", "Comprensión del fenómeno de la situación de calle", "Herramientas para abordar el fenómeno de la situación de calle", "Comprensión del rol de las condiciones para apoyar la intervención" y "Contribuciones específicas para mi formación en Trabajo Social". Cada uno de estos ítems representó afirmaciones con respecto a la práctica pre-profesional y los aprendizajes intencionados para la formación en Trabajo Social. Estos se midieron utilizando variables de intervalo en rangos de cero a diez. En cada caso, las personas encuestadas respondieron sobre el nivel de acuerdo y desacuerdo en el que la evaluación más baja (desacuerdo) correspondió a 0 y la más alta (acuerdo) a 10. Dado que las variables además eran de tipo continuo, las personas encuestadas pudieron utilizar decimales para responder el cuestionario. El análisis de los datos se guió por la estadística descriptiva. Asimismo, fue apoyado por SPSS 25. Para efectos de este artículo, se concentra la presentación de resultados utilizando medidas de tendencia central y de dispersión.

\section{Hallazgos}

\subsection{Apoyo recibido en la práctica pre-profesional}

En general, las personas encuestadas consideran que durante su práctica recibieron el apoyo de las instituciones en las que la desarrollaron, tanto en términos de guía u orientación técnica como de supervisión. Sin embargo, quienes se ubican en la categoría "Título profesional y Licenciatura en Trabajo Social" declaran, en promedio, que han tenido un mayor apoyo en comparación con aquellos que no tienen licenciatura. En este sentido, la mitad de quienes tienen una educación superior en Trabajo Social sin el grado de licenciado/a evalúa con 5 o menos, el apoyo recibido por la casa de estudios para acompañar su proceso. A su vez, en este ámbito y en la supervisión recibida de la institución de práctica, se observan personas que evalúan con 0 , lo que indicaría la presencia de casos que consideran que su práctica pre-profesional no habría supuesto una contribución con respecto al apoyo de la institución de educación superior y a la supervisión del centro de práctica. 


\subsection{Comprensión sobre la situación de calle}

Se observa una tendencia a considerar que la práctica contribuyó a comprender el fenómeno de la situación de calle, principalmente a través de la experiencia de las relaciones directas. El análisis de los valores mínimos muestra que, en relación con las variables revisadas en esta dimensión, hay casos de trabajadoras y trabajadores sociales que considerarían que su práctica pre-profesional no habría sido una contribución. Esto se muestra de manera más crítica en los ítems "comprensión del fenómeno de la situación de calle" y "comprensión de las lógicas de los programas sociales públicos para personas en situación de calle". Cabe señalar que esto es más crítico en la categoría con "Título Profesional y Licenciatura en Trabajo Social".

\subsection{Herramientas para la intervención}

Con respecto a la incorporación de herramientas para la intervención dentro del proceso de práctica pre-profesional -habiendo valores promedio en torno a 7 puntos- se aprecian valores de cero que dan cuenta de una baja valoración de su contribución al desarrollo de herramientas para diseñar intervenciones, implementar y evaluar los resultados de estrategias interventivas. Esto se aprecia de manera general en ambas categorías. Evaluación más alta se aprecia en torno a herramientas para participar en equipos multidisciplinarios y para discutir técnicamente y tomar decisiones. Asimismo, se evalúa, en términos generales de forma positiva, el haber podido incorporar herramientas para la generación y uso de información como insumo para implementar estrategias de intervención en situación de calle. Al igual que en las dimensiones anteriores, los valores mínimos corresponden a participantes que no reconocerían contribuciones sustantivas.

\subsection{Contribuciones específicas para la formación en Trabajo Social}

En cuanto a técnicas de autocuidado, no se observa necesariamente un alto reconocimiento de haber incorporado estrategias de auto cuidado en el proceso de práctica pre-profesional. En este sentido, se observa una visión más crítica en la categoría con licenciatura. Por su parte, en términos generales se evalúa positivamente el que el espacio de práctica pre-profesional habría contribuido a la formación en Trabajo Social a partir de la relación directa que se estableció con personas en situación de calle. En una línea similar, la práctica habría posibilitado desarrollar un cuestionamiento crítico de los principios éticos de la intervención en este ámbito. Asociado a esto, algunos participantes en el estudio considerarían que el proceso de práctica habría ayudado a comprender cómo manejar las diferencias dentro de la intervención y, en menor medida, a entender el enfoque de derechos en los procesos interventivos. Por su parte, con respecto a la evaluación de las principales habilidades psicosociales desarrolladas o potenciadas durante el proceso, hay una tendencia hacia una evaluación positiva. Esto igualmente se 
Andrade-Guzmán, y Eissmann-Araya

daría respecto a la comprensión del rol del Trabajo Social en la intervención en calle. En relación con el desarrollo de la reflexividad crítica para intervenir en este ámbito, igualmente, se observan en términos generales, altas evaluaciones. Finalmente, en esta dimensión, el análisis de valores mínimos muestra un grupo de casos que no reconocería contribuciones sustantivas de sus procesos de práctica pre-profesional.

\subsection{Comprensión sobre el rol de las condiciones de soporte a la intervención}

Finalmente, se observan en general evaluaciones promedio en torno a los 7,6 puntos con respecto a la comprensión del rol que cumplirían los recursos y las tecnologías para llevar adelante procesos de intervención en situación de calle. Los resultados respecto a cada variable se presentan en la Tabla 2. 
Tabla 2. Resultados

\begin{tabular}{|c|c|c|c|c|c|c|c|c|c|c|c|c|}
\hline & \multicolumn{4}{|c|}{$\begin{array}{l}\text { Título profesional y } \\
\text { Licenciatura }\end{array}$} & \multicolumn{4}{|c|}{$\begin{array}{l}\text { Educación Profesional o Técnica } \\
\text { (sin Licenciatura) }\end{array}$} & \multicolumn{4}{|c|}{ Total } \\
\hline & $\begin{array}{l}\text { Media } \\
\text { (DS2) }\end{array}$ & Mediana & Máx & Mín & $\begin{array}{c}\text { Media } \\
\text { (DS) }\end{array}$ & Mediana & Máx & Mín & Media (DS) & Mediana & Máx & Mín \\
\hline \multicolumn{13}{|l|}{ Apoyo recibido en la práctica pre-profesional } \\
\hline Apoyo académico recibido de la casa de estudios & $8.3(2.1)$ & 8.9 & 10.0 & 0.0 & $5.8(3.4)$ & 5.0 & 10.0 & 0.0 & $7.5(2.8)$ & 8.0 & 10.0 & 0.0 \\
\hline $\begin{array}{l}\text { Apoyo técnico del lugar de práctica (de } \\
\text { profesionales, otros) }\end{array}$ & $8.3(1.9)$ & 9.0 & 10.0 & 2.0 & $7.8(2.6)$ & 9.0 & 10.0 & 3.0 & $8.1(2.2)$ & 9.0 & 10.0 & 2.0 \\
\hline Supervisión del lugar de práctica pre-profesional & $8.6(2.0)$ & 10.0 & 10.0 & 2.0 & $7.6(3.1)$ & 9.5 & 10.0 & 0.0 & $8.3(2.4)$ & 10.0 & 10.0 & 0.0 \\
\hline \multicolumn{13}{|l|}{ Comprensión sobre la situación de calle } \\
\hline Comprensión del fenómeno de la situación de calle & $8.9(1.8)$ & 10.0 & 10.0 & 1.0 & $8.5(2.0)$ & 10.0 & 10.0 & 4.0 & $8.8(1.9)$ & 10.0 & 10.0 & 1.0 \\
\hline $\begin{array}{l}\text { Conocimiento directo de experiencias de vida que } \\
\text { ayudan a comprender el problema de la situación } \\
\text { de calle }\end{array}$ & $9.3(1.3)$ & 10.0 & 10.0 & 4.0 & $8.7(1.9)$ & 10.0 & 10.0 & 5.0 & $9.1(1.6)$ & 10.0 & 10.0 & 4.0 \\
\hline $\begin{array}{l}\text { Comprensión de las lógicas de los programas } \\
\text { sociales públicos para personas en situación de calle }\end{array}$ & $7.9(2.6)$ & 8.2 & 10.0 & 0.0 & $8.0(2.0)$ & 8.7 & 10.0 & 5.0 & $8.0(2.4)$ & 8.4 & 10.0 & 0.0 \\
\hline \multicolumn{13}{|l|}{ Herramientas para la intervención } \\
\hline $\begin{array}{l}\text { Herramientas para diseñar estrategias de } \\
\text { intervención }\end{array}$ & $7.7(2.2)$ & 8.0 & 10.0 & 0.0 & $7.8(2.8)$ & 8.0 & 10.0 & 0.0 & $7.7(2.4)$ & 8.0 & 10.0 & 0.0 \\
\hline $\begin{array}{l}\text { Herramientas para implementar estrategias de } \\
\text { intervención }\end{array}$ & $7.1(2.5)$ & 7.5 & 10.0 & 0.0 & $7.0(3.1)$ & 7.0 & 10.0 & 0.0 & $7.1(2.7)$ & 7.5 & 10.0 & 0.0 \\
\hline $\begin{array}{l}\text { Herramientas para evaluar los resultados de una } \\
\text { intervención }\end{array}$ & $7.0(2.6)$ & 8.0 & 10.0 & 0.0 & $7.0(2.9)$ & 8.0 & 10.0 & 0.0 & $7.0(2.7)$ & 8.0 & 10.0 & 0.0 \\
\hline $\begin{array}{l}\text { Herramientas para discutir técnicamente y tomar } \\
\text { decisiones }\end{array}$ & $8.0(2.2)$ & 9.0 & 10.0 & 2.0 & $7.5(2.5)$ & 8.0 & 10.0 & 2.0 & $7.9(2.3)$ & 8.5 & 10.0 & 2.0 \\
\hline $\begin{array}{l}\text { Herramientas para trabajar en equipos } \\
\text { multidisciplinares }\end{array}$ & $8.3(2.0)$ & 9.0 & 10.0 & 3.0 & $8.1(2.7)$ & 10.0 & 10.0 & 0.0 & $8.2(2.2)$ & 9.0 & 10.0 & 0.0 \\
\hline $\begin{array}{l}\text { Herramientas para generar y usar información en } \\
\text { los procesos de intervención }\end{array}$ & $8.0(2.5)$ & 9.0 & 10.0 & 0.0 & $7.6(2.3)$ & 8.0 & 10.0 & 3.0 & $7.8(2.5)$ & 8.8 & 10.0 & 0.0 \\
\hline
\end{tabular}

2 Refiere en todos los casos a desviación estándar 
Andrade-Guzmán, y Eissmann-Araya

\section{(Continuación)}

Título profesional y

Licenciatura

Media

(DS)
Educación Profesional o Técnica

(sin Licenciatura)

Media

(DS) Mediana Máx Mín
Total

Media

(DS)

\section{Contribuciones específicas para la formación en Trabajo Social}

Técnicas de auto-cuidado

Mediana Máx Mín

Experiencia de trabajo directo con personas en situación de calle

Espacio para revisar y cuestionar los principios éticos sobre la intervención

$\begin{array}{lccccccccccc}8.9(2.2) & 10.0 & 10.0 & 0.0 & 8.4(1.9) & 10.0 & 10.0 & 5.0 & 8.8(2.1) & 10.0 & 10.0 & 0.0 \\ 8.9(1.6) & 9.1 & 10.0 & 2.0 & 8.1(1,9) & 8.0 & 10.0 & 4.0 & 8.7(1.7) & 9.0 & 10.0 & 2.0 \\ 8.6(2.1) & 9.0 & 10.0 & 0.0 & 8.3(2.0) & 8.8 & 10.0 & 4.0 & 8.5(2.0) & 9.0 & 10.0 & 0.0 \\ 7.8(2.2) & 8.0 & 10.0 & 1.0 & 7.7(2.3) & 8.0 & 10.0 & 4.0 & 7.8(2.2) & 8.0 & 10.0 & 1.0 \\ 8.5(1.9) & 9.0 & 10.0 & 0.0 & 7.9(2.1) & 8.0 & 10.0 & 3.0 & 8.3(2.0) & 9.0 & 10.0 & 0.0 \\ 8.3(2.1) & 9.0 & 10.0 & 0.0 & 7.5(2.6) & 8.0 & 10.0 & 0.0 & 8.0(2.3) & 8.0 & 10.0 & 0.0 \\ 8.5(2.1) & 9.0 & 10.0 & 0.0 & 8.3(1.8) & 8.0 & 10.0 & 4.0 & 8.4(2.0) & 9.0 & 10.0 & 0.0\end{array}$

Comprensión de formas para trabajar con las diferencias/ diversidad

Comprensión de la forma en la que opera el enfoque de derechos en los procesos de intervención

Desarrollo de la reflexividad crítica para intervenir en situación de calle

Habilidades psicosociales para intervenir en situación de calle

Comprensión del rol del Trabajo Social en la intervención en calle

$9.0 \quad 10.0 \quad 0.0 \quad 7.5(2.2)$

8.0

$10.0 \quad 4.0 \quad 7.6(2.5)$

$\begin{array}{lll}8.5 & 10.0 \quad 0.0\end{array}$

Fuente: elaboración propia 


\section{Conclusiones}

Este estudio buscó responder el interrogante sobre cómo las y los trabajadores sociales chilenos evalúan los aprendizajes promovidos dentro de sus espacios de práctica pre-profesional para la intervención en el ámbito de la situación de calle. En este contexto, las evaluaciones de las personas encuestadas tienden a ser positivas con respecto a las herramientas incorporadas en estos espacios. Sin embargo, se observa la presencia de un grupo de profesionales de Trabajo Social que tiene una opinión más crítica de su experiencia. Así, los resultados muestran altos niveles de dispersión (al menos por rango) en las evaluaciones de los ítems sobre los cuales se les consultó. Este elemento es relevante, ya que si las y los trabajadores sociales evalúan en niveles muy bajos los resultados en torno a aprendizajes de sus experiencias de práctica pre-profesional, entonces existiría una base para afirmar que, al menos, en sus primeras experiencias de actuación profesional con personas en situación de calle, no todos sentirían que cuentan con las mejores herramientas para conducir y/o participar en estos procesos de intervención. Este tendería a ser el caso de quienes recibieron "Título profesional y Licenciatura en Trabajo Social", elemento que posibilita plantear la pregunta sobre si han percibido su práctica más bien centrada en tareas operativas y menos en el desarrollo de un enfoque reflexivo y crítico para contextualizar y problematizar la intervención en calle.

En general, estos resultados sugerirían la necesidad de reforzar las condiciones institucionales para llevar a cabo las prácticas pre-profesionales, con el fin de preparar a las y los trabajadores sociales con herramientas efectivas para abordar las iniciativas de transformación social en este ámbito de intervención. Este elemento dialoga con investigaciones previas que han relevado la necesidad de reforzar los contextos institucionales de enseñanza, para llevar a cabo procesos de transformación social (Varghese, 2016).Esto, sin embargo, no solo al nivel de las casas de estudio, sino también sobre las instituciones en donde se habría de desarrollar la práctica pre-profesional, reforzando especialmente, si bien no de forma exclusiva, la figura de la supervisión. En relación con este punto, se reconoce la retroalimentación constructiva como un mecanismo de mejora de los aprendizajes de quienes se encuentran estudiando, esto se ha destacado en investigaciones y trabajos anteriores(Andrews \& Harris, 2017; Beddoe et al., 2011; Kourgiantakis et al., 2019; Puig-Cruells, 2020). Sin embargo, en este estudio, hay quienes comparten que el apoyo de sus casas de estudio y de las instituciones no habría sido el mejor para llevar a cabo la práctica pre-profesional. Retomando la idea de que esta constituiría el corazón de la educación en Trabajo Social(Robertson, 2013), sería crucial entonces reforzarlas condiciones para que este componente educativo pueda, al máximo de sus posibilidades, operar como un espacio de formación que promueva la incorporación de aprendizajes positivos en quienes estudian Trabajo Social. 
Andrade-Guzmán, y Eissmann-Araya

Se advierten algunas limitaciones en este estudio. Primero, desde un punto de vista cuantitativo, el tipo de diseño muestral, al no ser probabilístico, limita el alcance de los resultados solo a la muestra utilizada. En este sentido, investigaciones futuras deberían intentar producir datos mediante un muestreo que apunte hacia la generalización estadística de los hallazgos. En relación con este punto, la muestra por conveniencia en este estudio logró producir principalmente información de personas que realizaron su práctica pre-profesional en el sector sin fines de lucro (94\%). En este sentido, solo el 6\% de las personas encuestadas realizó su práctica pre-profesional en gobiernos locales. Considerando este elemento, estudios futuros debiesen intentar incluir mayormente a personas que hayan realizado sus prácticas en el Estado, balanceando la presencia de informantes a nivel local, provincial, regional y central. Otra limitación de este trabajo está relacionada con la modalidad del instrumento. Con un cuestionario en línea, no es posible verificar el nivel de dedicación para responder las preguntas. En este marco, futuras investigaciones deberían realizar un trabajo de campo utilizando una encuesta cara a cara para monitorear este aspecto. Además, el alcance retrospectivo del instrumento, que invita a las personas encuestadas a responder preguntas relacionadas con procesos vividos en el pasado, es un elemento que aumenta la incertidumbre sobre el nivel de calidad de las respuestas. Nuevamente, futuras investigaciones debiesen intentar producir información sobre estudiantes de Trabajo Social que hayan recientemente finalizado su proceso para abordar en mejor medida este aspecto. Finalmente, esta investigación proporciona información valiosa sobre la evaluación dada a los aprendizajes intencionados en la práctica pre-profesional. Sin embargo, no exploró las posibles causas relacionadas con las diferencias en la evaluación dada a cada uno de los diferentes aspectos. En un futuro, se debería procurar explorar cuáles son las posibles razones de las diferencias, por ejemplo, en términos de tipo de educación recibida (con o sin licenciatura en Trabajo Social). Diseños cualitativos ayudarían sustantivamente en ello.

Cabe relevar que, pese a las limitaciones mencionadas, esta investigación ha proporcionado elementos valiosos sobre la educación en Trabajo Social y las experiencias de práctica pre-profesional en el ámbito de la intervención en situación de calle. En este sentido, esta investigación puede ser posiblemente uno de los pocos estudios que se han centrado en cómo antiguos estudiantes chilenos de Trabajo Social evalúan los aprendizajes incorporados en sus procesos de práctica en este ámbito de intervención específico. Por su parte, este estudio ha producido información de personas provenientes de diferentes tipos de formación en Trabajo Social en Chile, lo que aumenta la comprensión sobre este tipo de procesos desde una perspectiva más integral.

Diversas implicancias para una educación en Trabajo Social en el campo de la situación de calle desde una perspectiva crítica son reconocidas a partir de esta investigación. Primero, el hecho de que las evaluaciones presenten, en general, niveles 
Andrade-Guzmán, y Eissmann-Araya

altos de dispersión daría cuenta de la necesidad de mejorar los estándares de calidad de los procesos de educación superior. Un paso relevante en esta dirección sería homologar los elementos requeridos que cada persona debiese incorporar o reforzar, a partir de los procesos de práctica pre-profesional. Esto, en concordancia con el año de la formación. Al respecto, y desde la posición paradigmática que adopta este estudio, el Trabajo Social y la educación en Trabajo Social desde una perspectiva crítica deben estar siempre al servicio de los derechos. Desde esta perspectiva, esto implica asegurar umbrales mínimos de formación (Instituto de Políticas Públicas en Derechos Humanos del MERCOSUR-IPPDH, 2014; Sepúlveda, 2014)que se requerirían para la intervención en situación de calle. Asimismo, implica resguardar elementos mínimos para abordar iniciativas de promoción que contribuyan a modificar elementos estructurales que perpetúan la exclusión social de las personas afectadas por el fenómeno.

En este sentido, si hay trabajadores sociales que no sienten que obtienen los mejores aprendizajes de sus experiencias de práctica pre-profesional, es posible que en los procesos de intervención en los que ejercen como personas tituladas, al menos, durante sus primeros años de ejercicio, no cuenten con las mejores herramientas para facilitar una intervención transformadora. En este marco, estos elementos mínimos requeridos, en tanto estándares de calidad de una formación crítica y reflexiva, debería definirse participativamente y revisarse de forma continua para avanzar hacia la búsqueda constante de "máximos" en los procesos de formación en Trabajo Social.

Por su parte, los resultados de este estudio muestran la necesidad de problematizar la forma en que se contextualiza y comprende la intervención en situación de calle. Para avanzar en esta dirección, podría ser útil reforzar el papel de los procesos de inmersión, asegurando que quienes estudian puedan contar con personal experimentado para ayudarles a construir un diagnóstico situacional complejo. Además, este personal debería poder proporcionar las herramientas para comprender de manera crítica el rol de las personas en situación de calle, en las intervenciones guiadas desde un enfoque de derechos, y el papel de las personas profesionales en pos de asegurarles espacios para la participación activa en las intervenciones. Además, este personal es clave para promover una comprensión integral del rol de las políticas públicas, los responsables de la toma de decisión en calle, y otros actores en las intervenciones, así como el papel de los recursos en la actuación profesional en este ámbito de la intervención social.

Finalmente, incluso cuando hay una positiva evaluación general de los procesos de práctica pre-profesional en las personas encuestadas, las respuestas muestran una mayor evaluación de la supervisión y el apoyo proporcionado por la institución en donde esta fue llevada a cabo, respecto de la entregada por las casas de estudio. Basado en la relevancia de ambos actores en el proceso de educación superior, este elemento da cuenta de la necesidad de avanzar hacia el establecimiento y/o el refuerzo de una colaboración robusta 
Andrade-Guzmán, y Eissmann-Araya

entre ellos. Esto, revisando conjuntamente, antes de la inmersión en la práctica preprofesional, los propósitos de enseñanza a lograr a través de procesos. Concretamente, construyendo de forma conjunta formas de avanzar hacia ellos y definiendo el papel de cada esfera en los procesos, así como de generar y/o fortalecer mecanismos de aseguramiento de la calidad para facilitar el proceso formativo. Además, en una lógica de colaboración activa y de redistribución de poder, los estudiantes deberían tener una participación preponderante en la definición de, entre otros, los objetivos de aprendizaje, la generación de indicadores y la definición de estrategias y metodologías para lograrlos. Ello, generando espacios que contribuyan a la reflexión crítica de quienes estudian sobre su rol como practicantes y como futuros profesionales y/o técnicos del Trabajo Social.

\section{Referencias bibliográficas}

Al-Nashif, N. (2018). Human Rights and Social Work. Global Social Work, 8(ext.), 9-18. doi: 10.1016/B978-0-08-097086-8.28040-9.

Andrews, P., \& Harris, S. (2017). Using live supervision to teach counselling skills to social work students. Social Work Education, 5479, 1-13. doi: 10.1080/02615479.2016.1270929.

Aspeé, J. (2016). Análisis longitudinal de la exclusividad universitaria del Trabajo Social en Chile. Rumbos TS, (13), 10-32.

Beddoe, L., Ackroyd, J., Chinnery, S., \& Appleton, C. (2011). Live Supervision of Students in Field Placement: More than Just Watching Live Supervision of Students in Field Placement: More than Just Watching. Social Work Education, 30(5), 512-528. doi: 10.1080/02615479.2010.516358.

Belcher, J., Pecukonis, E., \& Knight, C. (2011). Where have all the teachers gone? The selling out of social work education. Journal of Teaching in Social Work, 31(2), 195-209. doi: $10.1080 / 08841233.2011 .562103$.

Birkenmaier, J., Wilson, R. J., Berg-Weger, M., Banks, R., \& Hartung, M. (2003). MSW Integrative Seminars: Toward Integrating Course and Field Work. Journal of Teaching in Social Work, 23(1-2), 167-182. doi: 10.1300/J067v23n01.

Calderón, M., y Cortés, R. (2014). Trabajo social en Chile. Una mirada a la formación en el escenario político y económico chileno. Revista de Treball Social, (202), 152-163.

Castañeda, P., y Salamé, A. (2003). Competencias profesionales de Trabajo Social: tradición y transformación. Revista de Trabajo Social, 72, 109-127.

Cea, M. (2001). Metodología Cuantitativa: Estrategias y técnicas de investigación social. Madrid, España: Editorial Síntesis.

Das, C., \& Carter, J. (2014). Strategies for critical reflection in international contexts for social work students. International Social Work, 57(2), 109-120. doi: $10.1177 / 0020872812443693$. 
Andrade-Guzmán, y Eissmann-Araya

Duarte, C., y Mora, A. (2016). Renovación curricular, una oportunidad para repensar el Trabajo Social en la Universidad de Atacama. Revista de Docencia Universitaria, 14(1), 203-223.

Eissmann, I., y Cuadra, C. (2018). El potencial de las Estrategias Residenciales para Superar la Situación de Calle: Aportes desde la Experiencia Chilena. Chile: Moviliza. Unidad de Investigación y Desarrollo. Recuperado de https://ighhub.org/sites/default/files/attachments/Reporte.Estudio\%20Residenci as $\% 20 y \% 20$ Casas $\% 20$ Compartidas.pdf.

Federación Europea de Organizaciones Nacionales-FEANTSA. (2016). Guía Housing First Europa. FEANTSA. Recuperado de https:/ / housingfirsteurope.eu/assets/files/2016/11/Gui\%CC\%81a-Housing-FirstEuropa.pdf.

Ghiraldelli, R. (2020). Formación Profesional y la Práctica Supervisada en el Servicio Social brasileño. Prospectiva. Revista de Trabajo Social e Intervención Social, (29), 39-56. doi: $10.25100 /$ prts.v0i29.7554.

González, M. (2010). “La visita de las moscas azules”. El concepto de “Visitación” como eje articulador de la formación de asistentes sociales en las primeras escuelas chilenas. Santiago, 1925-1935. En M. González (Ed.), Historias del Trabajo Social en Chile, 19252008. Contribución para nuevos relatos (pp. 23-51). Santiago de Chile: Ediciones Técnicas de Educación Superior.

Gorostiaga, J.M. (2017). La formación de investigadores en el campo de la política educativa: una mirada regional. Revista de la Educación Superior, 46(183), 37-45. doi: 10.1016/j.resu.2017.06.001.

Healy, K. (2001). Trabajo social: perspectivas contemporáneas. Madrid, España: Ediciones Morata.

Illanes, M. (2006). Cuerpo y sangre de la politica. La construcción histórica de las visitadoras sociales. Chile, 1887-1940. Santiago de Chile: LOM Ediciones.

Instituto de Políticas Públicas en Derechos Humanos del MERCOSUR-IPPDH. (2014). Ganar derechos. Lineamientos para la formulación de políticas públicas basadas en derechos. Recuperado de http://www.ippdh.mercosur.int/wpcontent/uploads/2014/12/GanarDerechos_Lineamientos1.pdf.

Iturrieta, S. (2017). Entre burbujas, sensaciones y realidades de la profesión más masificada en Chile: el Trabajo Social. Cuadernos de Trabajo Social, 1(9), 9-26.

Kourgiantakis, T., Sewell, K.M., \& Bogo, M. (2019). The Importance of Feedback in Preparing Social Work Students for Field Education. Clinical Social Work Journal, 47, 124-133. doi: $10.1007 /$ s10615-018-0671-8.

López, E., y Chaparro, M. (2006). Competencias laborales del trabajador social vistas desde el mercado laboral. Tabula Rasa, (5), 261-293.

ONU. Centro de Derechos Humanos. (1995). Derechos humanos y trabajo social. Manual para escuelas de servicio social y trabajadores sociales profesionales. Ginebra: Naciones Unidas. 
Andrade-Guzmán, y Eissmann-Araya

ONU. Consejo de Derechos Humanos. (2015). Informe de la Relatora Especial sobre una vivienda adecuada como elemento integrante del derecho a un nivel de vida adecuado y sobre el derecho de no discriminación a este respecto relativo a su misión a Cabo Verde, 23 Diciembre 2015, A/HRC/31/54/Add.1. Recuperado de https:/ / www.refworld.org.es/docid/56c57eb74.html.

Padget, D., Henwood, B., \& Tsemberis, S. (2016). Housing First Ending Homelessness, Transforming Systems, and Changing Lives. New York, United States: Oxford University Press.

Parola, R.N. (2020). Problematizando las prácticas preprofesionales en Trabajo Social. Desafíos y perspectivas. Prospectiva. Revista de Trabajo Social e Intervención Social, (29), 73-92. doi: $10.25100 /$ prts.v0i29.8714.

Puig-Cruells, C. (2020). El rol docente del tutor y supervisor de prácticas en Trabajo Social: construcción de la reflexividad y el compromiso durante la formación a través de la supervisión. Prospectiva. Revista de Trabajo Social e Intervención Social, (29), 57-72. doi: $10.25100 /$ prts.v0i29.8084.

Robertis de, C. (2018). Los derechos humanos, principios orientadores de la práctica del trabajo social. Global Social Work, 8(ext.), 19-34. doi: 10.30827/tsg-gsw.v8i0.7258.

Robertson, J.S. (2013). Addressing professional suitability in social work education: Results of a study of field education coordinators' experience. Journal of Practice Teaching $\mathcal{E}$ Learning, 11(3), 98-117. Recuperado dehttps://journals.whitingbirch.net/index.php/JPTS/article/view/278.

Rosas-Escamilla, R., y Maldonado-Maldonado, A. (2018). Los aprendices de brujos o los primeros acercamientos hacia la investigación. Un estudio sobre el programa del Verano de la Investigación Científica. Revista de La Educación Superior, 47(185), 33-56.

Rubilar, G. (2018). Trabajo Social y Derechos Humanos: perspectivas, posibilidades y desafíos a partir de la experiencia chilena. Global Social Work, 8(ext.), 120-144. doi: $10.30827 /$ tsg-gsw.v8i0.6494.

Salamé, A., y Castañeda, P. (octubre, 2009). Evolución de la formación profesional en Trabajo Social en Chile.En XIX Seminario Latinoamericano de Escuelas de Trabajo Social. El Trabajo Social en la coyuntura latinoamericana: desafíos para su formación, articulación y acción profesional. Universidad Católica Santiago de Guayaquil, Guayaquil, Ecuador. Recuperado de http:/ / www.ts.ucr.ac.cr/binarios/congresos/reg/slets/slets-019-315.pdf.

Saravia, F. (2015). La formación de los trabajadores sociales en Chile: ¿un asunto de oferta y demanda? Cuadernos de Trabajo Social, 28(1), 69-81. doi: $10.5209 /$ rev_cuts.2015.v28.n1.46510.

Sepúlveda, M. (2014). De la retórica a la práctica: el enfoque de derechos en la protección social en América Latina. Santiago de Chile: Comisión Económica para América Latina y el Caribe (CEPAL). 
Andrade-Guzmán, y Eissmann-Araya

Shera, W., \& Bogo, M. (2001). Social Work Education and Practice. Planning for the future. International Social Work, 44(2), 197-210.

Staub-Bernasconi, S. (2016). Social Work and Human Rights - Linking Two Traditions of Human Rights in Social Work. Journal of Human Rights and Social Work, 1(1), 40-49. doi: 10.1007/s41134-016-0005-0.

Toledo, U. (2004). ¿Una Epistemología del Trabajo Social? Cinta Moebio, 21(1), 200-214.

Us Department of housing and urban development office of policy development and research. (2010). Life After Transitional Housing for Homeless Families. Recuperado de https://www.huduser.gov/portal/publications/LifeAfterTransition.pdf.

Varghese, R. (2016). Teaching to Transform? Addressing Race and Racism in the Teaching of Clinical Social Work Practice. Journal of Social Work Education, 52(1), S134-S147. doi: 10.1080/10437797.2016.1174646.

Vidal, P. (2015). La educación del trabajo social en Chile: hacia un siglo de historia. Quaderni Del Csal, 3, 177-192.

Wayne, J., Raskin, M., \& Bogo, M. (2010). Field education as the signature pedagogy of social work education. Journal of Social Work Education, 46(3), 327-339. doi: 10.5175/JSWE.2010.200900043. 


\section{OTROS ARTÍCULOS DE PROSPECTIVA No. 30 DE 2020}

\section{EDITORIAL}

Coherencia, integridad y vida cotidiana

Luz Mary Sánchez-Rengifo

\section{ARTÍCULOS}

Trabajo Social y medios de comunicación: perspectivas y posibilidades de encuentro Social

Mercedes Muriel-Saiz

Maribel Martín-Estalayo

Seguridad, afectos y familias. Obstáculos en el proceso de reintegración de personas desmovilizadas de grupos

armados en Santander, Colombia

Jakeline Vargas-Parra

Ángela María Díaz-Pérez

Priscyll Anctil-Avoine

Reflexividad sobre la intervención profesional en duelo con población afectada por el conflicto armado en Colombia

María Cénide Escobar-Serrano

Maritza Charry-Higuera

Natalia Ramírez-Moncada

Ser mujer indígena, náhuatl, casada, migrante, sin trabajo remunerado: una realidad en los albergues jornaleros agrícolas en Colima, México

Nancy Elizabeth Molina-Rodríguez

Tipos y manifestaciones de la violencia de género: una visibilización a partir de relatos de mujeres víctimas en Soacha, Colombia

Diana Carolina Tibaná-Ríos

Diana Alejandra Arciniegas-Ramírez

Ingrid Julieth Delgado-Hernández

Análisis de necesidades en familias monoparentales con jefatura femenina usuarias de servicios sociales de atención primaria en España

Celia María Fernández-Martínez

Manuela Avilés-Hernández

Experiencias de intervención de trabajadoras sociales con trabajadores sexuales masculinos en Bogotá, Colombia Johan Arturo Barrera-Castellanos
Condiciones laborales de trabajadores sociales en hospitales públicos en la provincia de Mendoza,

Argentina

Cecilia Amalia Molina

Yanina Noemi Roslan-Angeloni

Analía Graciela Correa

Viviana Elena Varela

Gubernamentalidad neoliberal: miradas desde las intervenciones del Trabajo Social en el Gran La Plata, Argentina

Paula Mara Danel

Marcela Claudia Velurtas

Agustina María Favero-Avico

Educación superior en Trabajo Social en Chile y formación para la intervención en situación de calle. Desafios desde la evaluación que interventores hacen de sus procesos de práctica pre-profesional

Carlos Alejandro Andrade-Guzmán

Ignacio Andrés Eissmann-Araya

Educación en Derechos Humanos para el Trabajo Social en Chile: una mirada desde los estándares

internacionales

Lury Soledad Reyes-Pérez

Vivianne Soledad Hasse-Riquelme

Luis Marcelo Silva-Burgos

Arriesgar y preservar la vida: derechos humanos, conflicto sociopolítico armado y Trabajo Social en Colombia

Cristian Sebastián Castaño-Orozco

Ricardo Patiño-Martínez

\section{IN MEMORIAM}

Cristina Bautista Taquinás. Mujer indígena Nasa, Trabajadora Social, lideresa del norte del Cauca, Colombia

Alba Nubia Rodríguez-Pizarro

Lady Johanna Betancourt-Maldonado
ARTISTA INVITADO
MALA JUNTA KLAN
Alejandra Gutiérrez-Cárdenas

\section{PROSPECTIVA}

